\title{
MALIGNANT SERTOLI CELL TUMOUR: AN UNCOMMON TESTICULAR TUMOUR
}

\author{
Sia $S F^{\prime}$, Dublin $N^{\prime}$, Nurul $B^{2}$ and Wong $K T^{2}$ \\ Department of Surgery, Faculty of Medicine, University of Malaya, 50603 Kuala Lumpur, Malaysia \\ ${ }^{2}$ Department of Pathology, Faculty of Medicine, University of Malaya, 50603 Kuala Lumpur, Malaysia
}

\begin{abstract}
We report a case of an 86 year old Chinese man who presented with a painless right testicular swelling that had persisted for one year. There was no history of maldescend or cryptorchid testes. Clinical and ultrasound examination revealed testicular tumour with two round masses within the right scrotal sac, with minimal fluid seen within the sac. Tumour markers were normal. He subsequently underwent a right inguinal orchidectomy under local anaesthesia as he had an underlying cardiac insufficiency. Histopathological examination revealed malignant Sertoli cell tumour. True Sertoli cell mesenchyme tumours constitute less than $1 \%$ of all testicular cancers. Current literature on histopathological and clinical features and treatment options are reviewed. (JUMMEC 2006; 9(2): 18-21)
\end{abstract}

KEYWORDS: Malignant Sertoli cell tumours

\section{Introduction}

Sertoli cell tumour is a rare testicular stromal neoplasm in adults and constitutes less than $1 \%$ of all testicular tumours. It is usually benign (I), and only approximately $10 \%$ are malignant. However, it is among the most common testicular neoplasms in canines (2). In humans, more than 130 cases of malignant Sertoli cell tumours have been reported worldwide (2). We report the first case of malignant Sertoli cell tumour of the testis in Malaysia.

\section{Case Report}

An 86 yearold Chinese man with underlying ischaemic heart disease presented with painless right testicular swelling. The swelling had been present for one year. There was no history of maldescend or cryptorchid testes. He denied testicular or groin pain, fever, chills, weakness, weight loss or other constitutional symptoms. Physical examination revealed an enlarged right testicle. There was no gynaecomastia. Ultrasound examination revealed a testicular tumour with two round hyperechoic masses within the right scrotal sac with minimal hydrocele. Serum levels of $\alpha$-fetoprotein, $\beta$-human chorionic gonadotropin and lactate dehydrogenase were normal. After taking his underlying cardiac insufficiency into consideration, he underwent right inguinal orchidectomy under local anaesthesia.

\section{Histopathology}

Grossly, the testis measured $6 \mathrm{~cm} \times 3.5 \mathrm{~cm} \times 3 \mathrm{~cm}$, and the haemorrhagic epididymis was approximately $3.5 \mathrm{~cm}$ in length. Cut section of the testis showed a multiloculated cystic structure containing serous fluid, measuring $2 \mathrm{~cm} \times 1.8 \mathrm{~cm} \times 1.5 \mathrm{~cm}$ and an adjacent well circumscribed firm yellowish lesion measuring $3 \mathrm{~cm} \times$ $3 \mathrm{~cm} \times 2.5 \mathrm{~cm}$. Both the lesions were within the tunica albuginea (Figure I).

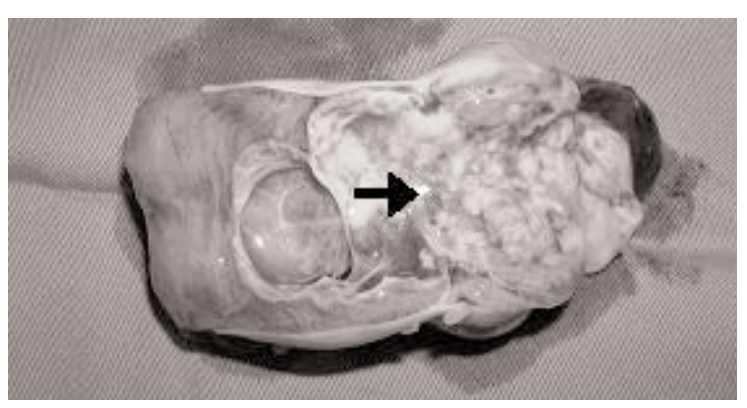

Figure I. Multiloculated cystic with firm mass (arrow) in orchidectomy specimen

Correspondence:

Dr Sia SF

Department of Surgery

Faculty of Medicine

University of Malaya

50603 Kuala Lumpur, Malaysia

Tel: 603-7949 3852

Email: sfsia02@um.edu.my 
Histological sections showed that the yellowish mass consisted of sheets of tumour cells exhibiting mainly tubule formation and an alveolar like pattern. The tumour cells were large, polyhedral, with small hyperchromatic nuclei and clear cytoplasms which contained fat (as demonstrated by oil red $O$ stain) (Figure 2). There was little glycogen within the cytoplasm. Mitosis and necrosis were present (Figure 3). In addition some papillary areas were noted. There was infiltration by neutrophils. No hyaline globules were identified and immunohistochemistry for $\alpha$ - fetoprotein was negative. Although this tumour was largely encapsulated, there was one area of capsular and vascular invasion (Figure 4).

The spermatic cord (surgical margin) was free of tumour. A section from the cyst wall showed thick mature fibrous tissue mainly devoid of lining epithelium and some areas were lined with single layer cuboidal cells. Inactive seminiferous and hyalinized/ fibrosed tubules were present outside the cyst wall. No in situ malignant change was noted.

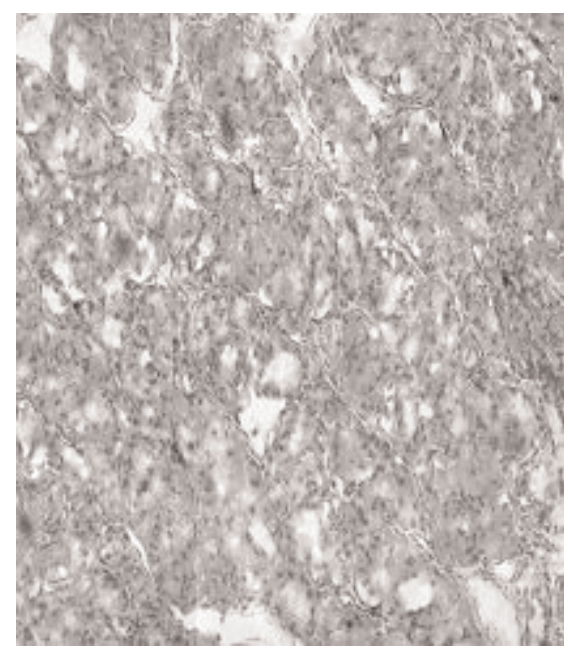

Figure 2. Shows large, polyhedral cells with small hyperchromatic nuclei and clear cytoplasm which contain fat (oil red $O$ stain: original magnification $\times 40$ )

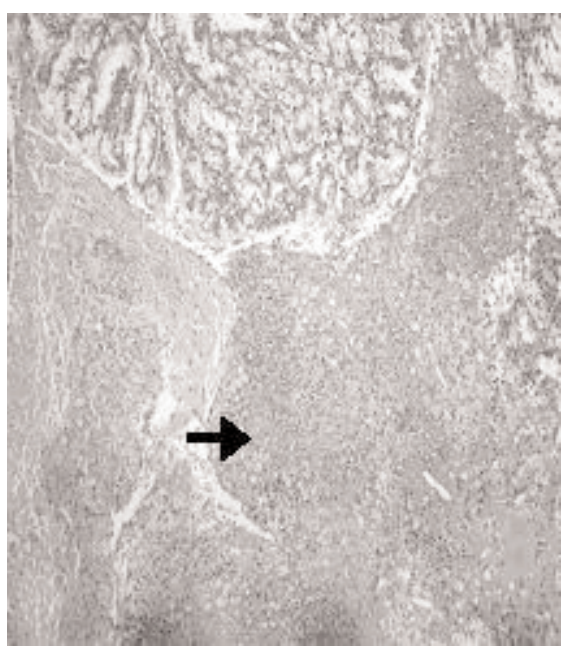

Figure 3. Shows area of necrosis (arrow) (Haematoxylin and eosin stain: original magnification $\times 10$ )

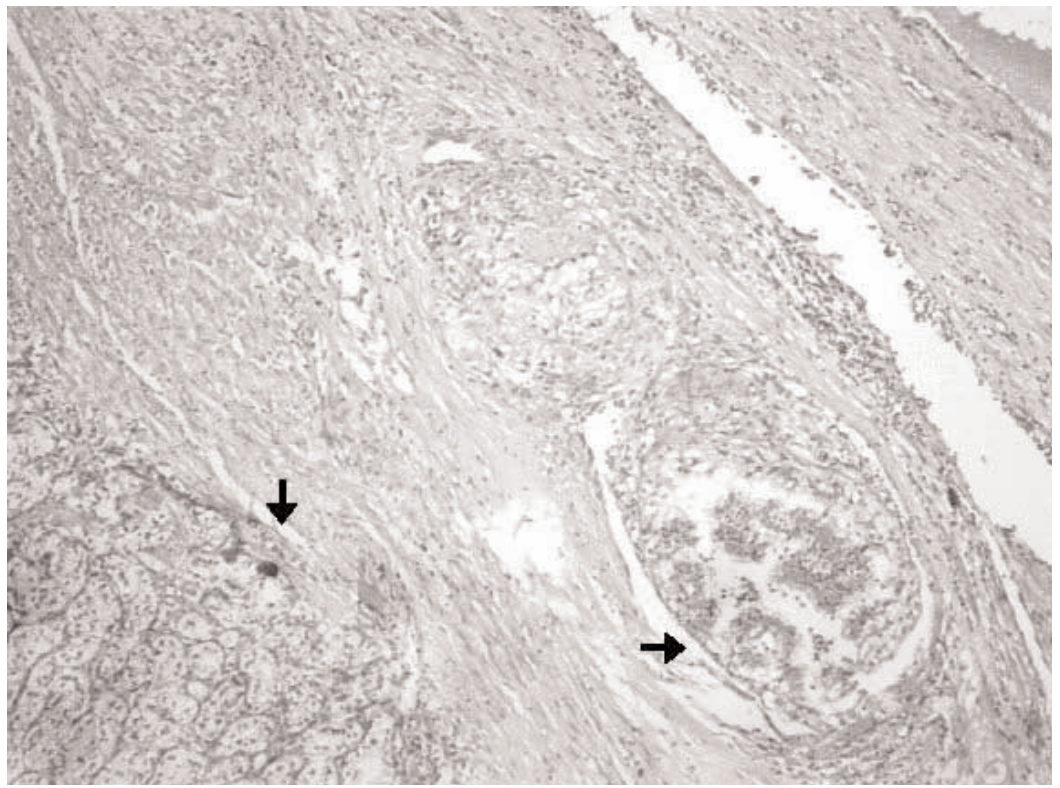

Figure 4. Shows area of capsular and vascular invasion (arrow) (Haematoxylin and eosin stain: original magnification $\times 10$ ) 


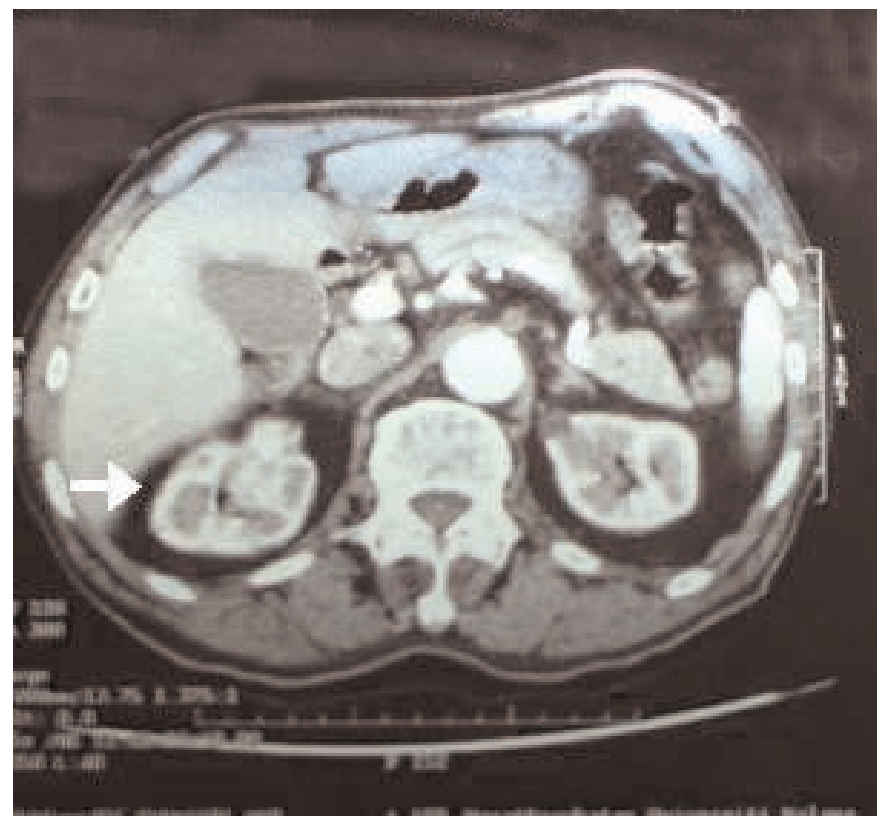

Figure 5. CT scan shows ill-defined mass at right kidney (white arrow)

Computerised tomography of the chest, abdomen and pelvis did not reveal any presence of mediastinal, paraortic or pelvic lymphadenopathy. The liver, spleen, pancreas and left kidney were normal. However, the right kidney appeared irregular in shape with an ill-defined mass seen at the right mid and upper pole measuring $2.9 \mathrm{~cm} \times 2.2 \mathrm{~cm} \times 2.5 \mathrm{~cm}$ (Figure 5).

As the general condition of the patient was poor, the family declined further treatment. No further investigations were performed, although it was speculated that the right kidney was involved by the Sertoli cell tumour.

\section{Discussion}

Sertoli cell tumours also known as androblastoma or gonadal stromal tumours, are rare testicular stromal neoplasm and comprise $0.4-1.5 \%$ of all testicular tumours (3). Patients with malignant Sertoli cell tumours show a wide age range, similar to that observed in patients with benign Sertoli cell tumours. Sertoli cell tumours have been reported in newborns $(2,3)$ and in patients as old as the octogenarian in the present case. Mean age of these patients was reported as 39 years old (4).
Most patients present with painless testicular swelling. These tumours occur with equal frequency in either testes, and are occasionally associated with cryptochid or maldescend testes. Almost $30 \%$ of reported cases have been associated with gynaecomastia (5). It has also been reported that the majority of cases were hormonal inactive and laboratory hormonal assays were inconsistent (3). Eble et al (4) reported that tumour size varies, and that size is not a reliable indicator of tumour behaviour or prognosis.

Diagnosis by fine needle aspirate has been proposed (6). However, this is not normal practice and the authors do not favour this method as there is a risk of cutaneous dissemination. The only absolute criterion for the diagnosis of malignancy is made by histopathology of the orchidectomy specimen along with the demonstration of metastasis. The diagnosis of this tumour can be difficult, frequently misinterpreted as seminoma (7).

The basic pathological pattern is of cells resembling normal Sertoli cells arranged in the nests and tubules, with or without lumina. Mitotic figures are occasionally present, and are associated with poorer prognosis. Three distinct patterns have been reported, (a) well 
demarcated nodules (b) replacement of the entire testis and (c) infiltration (4).

In the present case, malignancy was diagnosed on the basis of microscopic features, namely the combination of mitosis, necrosis and evidence of local vascular invasion.

Sertoli cell tumours commonly metastasize via the lymphatic system. Haematogenous spread to the lung, bone, pleura, thigh and adrenal gland has been reported (5). In a large series of Sertoli cell tumours, no synchronous renal mass or renal metastasize were reported. In this case the renal pathology could have been a metastatic deposit or a synchronous renal cell carcinoma. A renal biopsy would have been useful.

Radical inguinal orchidectomy remains the mainstay of treatment. Retroperitoneal lymph node dissection and adjuvant radiation therapy have been advocated to offer the best result (4), however, results of chemotherapy have been disappointing. These tumours may recur years after the ochidectomy, therefore long term follow-up is recommended (8).

\section{References}

I. Peterson RO. Testis in Urologic Pathology. Philadelphia: JB Lippincott 1992; 487-49।.

2. Talerman A. Malignant sertoli cell tumour of the testes. Cancer 1971; 28(2): 446-455.

3. Kolon TF, Hochman HI. Malignant sertoli tumour in a prepubescent boy. J Urol 1997; I58(2): 608-609.

4. Eble JN, Hull MT, Warfel KA. Malignant sex cord stromal tumour of testes. J Urol I984; I3 I (3): 546-550.

5. Hopkins GB, Parry HD. Metastasizing sertoli cell tumour (Androblastoma). Cancer 1969; 23(2): 463-467.

6. Nijhawan R, Dey P, Savitri K, et al. Fine needle aspiration cytology in malignant sertoli cell tumours of the testes. A case report. Acta Cytol. 1992; 36(4): 563-565.

7. Henley JD, Young RH, Ulbright TM. Malignant sertoli cell tumours of the testes: a study of 13 examples of a neoplasm frequently misinterpreted as seminoma. Am J Surg Pathol. 2002; 26(5): 54I-550.

8. Herrera LO, Wilk H, Wills JS, et al. Malignant (Androblastoma) sertoli cell tumours of testes. Urology 1981; 18(3): 287-290. 